\title{
EXPERIMENTAL RATIONALE OF THE USE OF CELL THERAPY FOR THE TREATMENT OF GLAUCOMA OPTICAL NEUROPATHY
}

\author{
Sergiy Rykov ${ }^{1}$ \\ eye-bolit@ukr.net \\ Oksana Petrenko \\ visionpetrenko@gmail.com \\ Antonina Yakovets \\ dr.antonina.yakovets@gmail.com \\ Dmytro Zubov \\ zoubov77@yahoo.com \\ Roman Vasyliev ${ }^{2}$ \\ romanvasyliev8@gmail.com \\ ${ }^{1}$ Department of Ophthalmology \\ Shupyk National Medical Academy of Postgraduate Education \\ 9 Dorohozhytska str., Kyiv, Ukraine, 04112 \\ ${ }^{2}$ Department of Genetic Diagnostics \\ State Institute of Genetic and Regenerative Medicine NAMS of Ukraine \\ 67 Vyshgorodska str., Kyiv, Ukraine, 04114
}

\begin{abstract} thalmology.

Development of new effective treatments for glaucomatous optic neuropathy is one of the most acute aspects of modern oph-

The aim of the work is to investigate the effectiveness of cell therapy with postnatal multipotent neural crest stem cells (NCSCs) using different cell delivery methods in a model of adrenaline-induced glaucoma.

Materials and methods. Glaucoma was induced in Wistar rats by intraperitoneal injections of $10 \mu \mathrm{g}$ to $15 \mu \mathrm{g} / 100 \mathrm{~g}$ body weight of $0.18 \%$ adrenaline hydrotartrate. NCSCs were delivered intravenously ( 5 million cells), retrobulbarly ( 0.5 million cells) or parabulbarly ( 0.5 million cells). Histomorphometric analysis of the retina was performed on stained haematoxylin-eosin sections with a thickness of $5 \mu \mathrm{m}$ one month after the delivery of NCSCs.

Results. NCSCs transplantation by all modes of delivery caused positive morphological changes to varying degrees. Intravenous administration induced a decrease in edema in all retinal layers and a slight restoration of the cytoarchitectonics of the retinal layers. The parabulbar administration of NCSCs led to a decrease in edema and the restoration of the cytoarchitectonics of the layers, most pronouncedly the ganglion cell layer and the inner retinal layer. After the retrobulbar administration of NCSCs, the reduction in edema and restoration of the cytoarchitectonics of the layers were the most pronounced.

Conclusions. According to the results of the study, the positive effect of NCSCs transplantation in an experimental model of glaucoma was the most pronounced following the retrobulbar injection of cells. Further investigations of the mechanisms of the effect of transplanted NCSCs on retinal structure restoration are needed.
\end{abstract}

Keywords: glaucoma, multipotent stem cells, cell therapy.

DOI: $10.21303 / 2504-5679.2020 .001187$

\section{Introduction}

Glaucoma is one of the leading causes of blindness and disability worldwide $[1,2]$. According to an epidemiological study, the number of people with glaucoma will increase by $74 \%$ from 2013 to 2040 [3]

Glaucoma is defined as progressive optic neuropathy characterized by the excitation of the optic disc and a corresponding decrease in retinal sensitivity [1, 4]. Glaucoma optic neuropathy 
involves the progressive loss of retinal ganglion cells and their axons, the induction of which is influenced by both external and internal factors [5, 6].

Many experimental studies using modern science techniques have been carried out, and it has been shown that multipotent stem cells have a positive effect in ophthalmology [7]. Advances in regenerative medicine and cell therapy are based on the unique properties of stem cells, including their ability to self-renew and differentiate into specific cell types, and the prospects for clinical use are virtually limitless [8-10].

Given the steady increase in the morbidity of glaucoma and the associated progressive deterioration and loss of visual function, which leads to disability and disability, glaucoma is a socially significant disease Thus, developing new effective treatments for glaucoma is one of the most pressing challenges in modern ophthalmology. A promising strategy for the treatment of glaucoma is cell therapy using stem cells, but an important consideration is determining the appropriate cell type.

In our study, we focused on multipotent neural crest stem cells (NCSCs). Several studies have shown that NCSCs are able to transdifferentiate into CNS cell types both in vitro and after in vivo transplantation [11-13]. In recent years, postnatal NCSCs has been identified in a number of tissues and organs of adult mammals, including humans. Thus, NCSCs demonstrate 2 basic properties of stem cells (SCs): the ability to self-renew and the potential for multilinear differentiation [14].

The goal of this work was to investigate the effectiveness of cell therapy with postnatal multipotent neural crest stem-derived stem cells using various delivery methods for the treatment of adrenaline-induced glaucoma.

\section{Materials and methods}

In experimental work, there were 50 Wistar rats (10-12 months, male). Laboratory animals were divided into 5 groups: intact animals group $(n=10)$, glaucoma-modelling group without NCSCs $(n=10)$, glaucoma-modelling group with intravenous injections of NCSCs $(n=10)$, glaucoma-modelling group with parabulbar injection of NCSCs $(n=10)$, glaucoma-modelling group with retrobulbar injection of NCSCs $(n=10)$.

Glaucoma was induced by intraperitoneal injections of $10 \mu \mathrm{g}$ to $15 \mu \mathrm{g} / 100 \mathrm{~g}$ body weight of $0.18 \%$ adrenaline hydrotartrate. Twenty injections were administered over 6 weeks, with an average of 3 injections per week. After every 5 injections, the dose of injected adrenaline was increased [15]. Intraocular pressure (IOP) was measured with an applanation tonometer (Icare ${ }^{\circledR}$ TONOVET tonometer, Finland).

During the experiment, bioethical principles, legislation and biomedical research requirements, namely, the Declaration of Helsinki (1964-2013), the Constitution of Ukraine and the Civil Code of Ukraine (2006), the Basics of the Legislation of Ukraine on Health Care (1992), and the Guidelines of Clinical Research of the Ministry of Health of Ukraine No. 42-7.0: 2005 "Medicines. Good Clinical Practice" (2005), Model Regulations on Ethics Commissions for Clinical Trials (Order of the Ministry of Health of Ukraine No. 690 of 23-9.2009), Law of Ukraine No. 3447-IV “On protection of animals from ill-treatment" (2006) were observed.

Rat NCSCs were obtained from hair follicles by vibration and cultured according to a previously described method [16]. Rat NCSCs were characterized by immunofluorescence analysis. NCSCs were transplanted intravenously ( 5 million cells in $0.5 \mathrm{ml}$ of saline), retrobulbarly ( 0.5 million cells in $0.05 \mathrm{ml}$ of saline) or parabulbarly ( 0.5 million cells in $0.05 \mathrm{ml}$ of saline). Histomorphometric analysis of the retina and optic nerve was performed on haematoxylin-eosin stained sections with a thickness of $5 \mu \mathrm{m}$.

\section{Results}

Rat NCSCs expressed markers characteristic of cells originating from the neural crest, namely, the transcription factor Sox10 and the intermediate filament protein Nestin (Fig. 1).

The IOP of rats prior to the start of glaucoma modelling was 7-8 $\mathrm{mm} \mathrm{Hg}$. Histological preparations of the retinas of intact rats are presented in Fig. 2. After glaucoma modelling, the IOP was 20-22 mm Hg. One month after glaucoma modelling, histological specimens exhibited glaucoma optic neuropathy, specifically swelling of all retinal layers; axonal degeneration in the 
nerve fibre layer; partial detachment from the ganglion cell layer; apoptosis and cell necrosis in the ganglion cell layer; loosening and increased thickness of the inner mesh layer and the appearance of cell nuclei; thinning and breaking of the outer mesh layer and the appearance of cell nuclei; the disruption of cytoarchitectonics in the inner and outer nuclear layers; a reduction in the number of pycnotic nuclei in these layers; increased thickness of the photoreceptor layer; and stasis and haemorrhage in the choroid (Fig. 3).

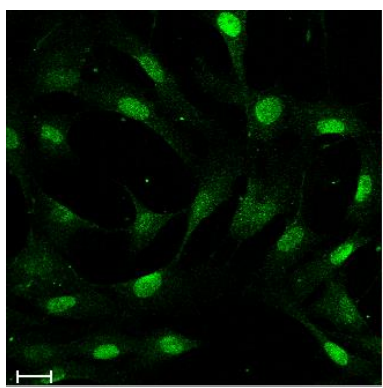

$a$

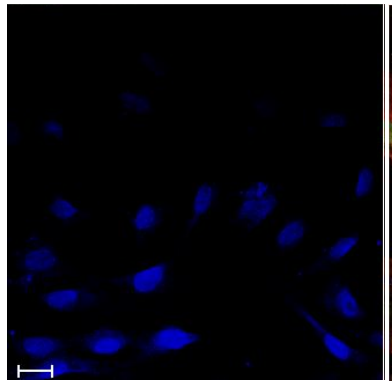

c

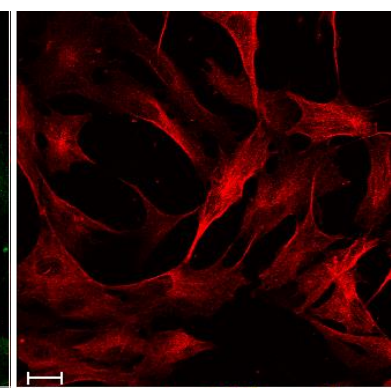

$b$

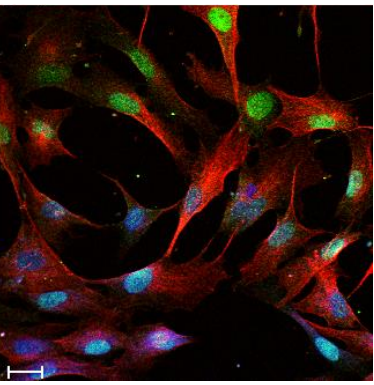

d

Fig. 1. Immunofluorescence analysis of NCSCs in rats: $a$-detection of the transcription factor Sox 10 (green); $b$ - detection of the intermediate filament protein Nestin (red); $c$ - hoechst 33342 staining of cell nuclei (blue); $d$-merged image. Scale bar=20 microns.

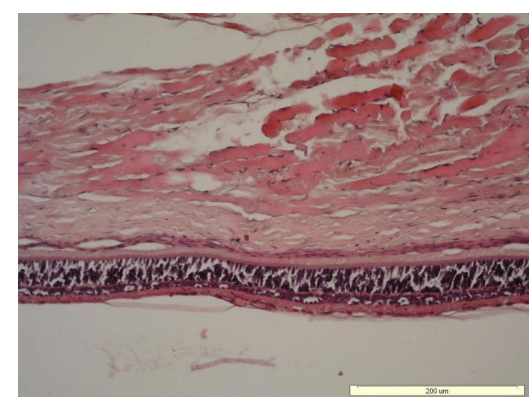

Fig. 2. Histological preparations of the retinas of intact rats. Haematoxylin-eosin staining

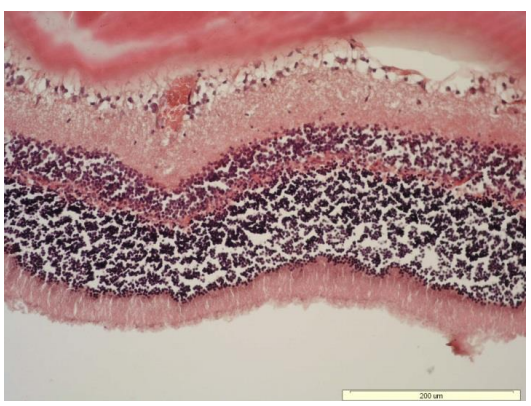

Fig. 3. Histological preparations of retinas after glaucoma modelling. Haematoxylin-eosin staining 
According to the results of the study, NCSCs transplantation by all modes of delivery caused positive morphological changes in the retina to varying degrees. Intravenous administration reduced the swelling of all retinal layers but did little to restore the cytoarchitectonics of the retinal layers (Fig. 4). Parabulbar administration of NCSCs led to a decrease in edema and the restoration of the cytoarchitectonics of the layers, most pronouncedly the ganglion cell layer and the inner retinal layer. Significant edema was maintained in the outer nuclear and photoreceptor layers (Fig. 5).

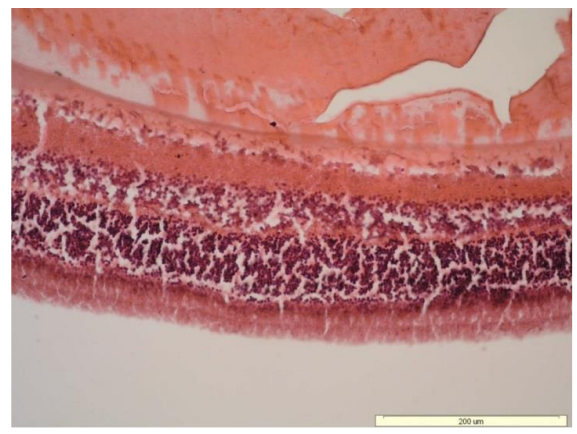

Fig. 4. Histological preparations of the retinas of glaucoma model rats injected intravenously with cells. Haematoxylin-eosin staining

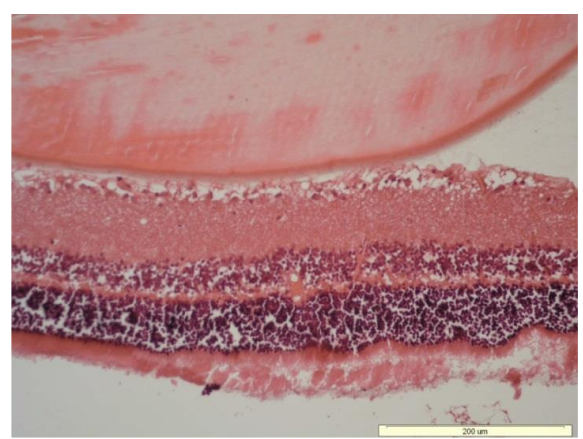

Fig. 5. Histological preparations of the retinas of glaucoma model rats injected parabulbarly with cells. Haematoxylin-eosin staining

After the retrobulbar administration of NCSCs, the reduction in edema and the restoration of the cytoarchitectonics of the layers were the most pronounced (Fig. 6).

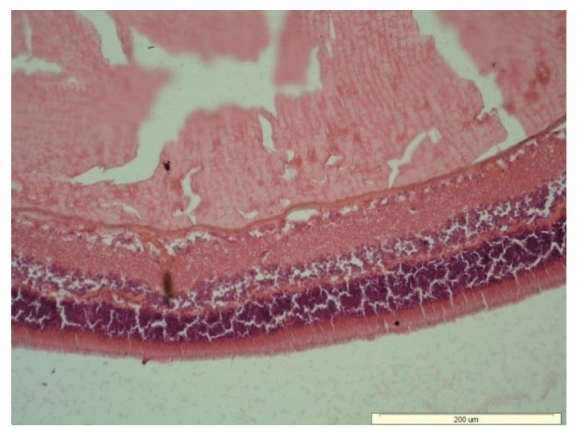

Fig. 6. Histological preparations of the rat retinas of glaucoma rats injected retrobulbarly with cells. Haematoxylin-eosin staining

\section{Discussion}

The number of glaucoma patients is increasing, and glaucoma is one of the most debilitating diseases and one of the leading causes of blindness worldwide [3]; thus, the development of new 
effective treatments for glaucoma is one of the pressing challenges of modern ophthalmology. A promising strategy is cell therapy using stem cells.

An important consideration of cell therapy is choosing the appropriate cell type. In our study, we focused on multipotent neural crest stem cells (NCSCs). This is because of the easy accessibility of these cells, the minimally invasive procedure required to obtain them, and their biological properties, namely, their potential for multilinear differentiation and their ability to produce a wide range of growth factors and cytokines with neurotrophic, neuroprotective, anti-angiogenic and apoptotic effects [17].

With respect to the biological properties of NCSCs, it should be noted that they have the potential to differentiate into major cell types, namely, nerve crest derivatives such as neurons and glia of the peripheral nervous system, melanocytes, stromal cells, corneal endothelial cells, and some types of endocrine cells [14]. Importantly, there is experimental evidence that NCSCs are capable of linear conversion into CNS neuronal stem cells [11]. In addition, NCSCs can transdifferentiate into CNS cell types, such as oligodendrocytes, after transplantation into a new microenvironment [13]. This provides a basis for studying the possible transdifferentiation of NCSCs into retinal ganglion cells and photoreceptor cells. In addition to potentially being able to directly integrate into damaged tissues, NCSCs can also realize their regenerative potential through paracrine action. Thus, they have been found to produce a wide range of neurotrophic factors (brain-derived neurotrophic factor (BDNF), glial-derived neurotrophic factor (GDNF), neurotrophin 3, neurotrophin 4/5 and nerve growth factor (NGF)) and growth factors (the major fibroblast growth factor FG, vascular endothelial growth factor (VEGF), etc.), which determines their neuroprotective and regenerative potential. In summary, these properties make postnatal NCSCs promising cells for the development of new treatments for diseases of the cornea, retina, and optic nerve $[18,19]$.

To date, numerous studies have shown that the use of cell therapy for the treatment of experimental glaucoma has a positive effect, but the results are controversial and not consistent $[8,9,20]$.

NCSCs demonstrate 2 basic properties of SCs: the ability to self-renew and the potential for multilinear differentiation. A very promising source for the isolation and cultivation of NCSCs from animals and humans are hair follicles, and the procedure for obtaining a small skin biopsy specimen from a patient is minimally invasive [16].

Our study demonstrates that cell therapy with NCSCs through all delivery methods used has a positive effect on retinal structure. One month after the intravenous delivery of NCSCs, there was a significant decrease in edema and partial restoration of the structure of all layers of the retina, and after parabulbar administration, these positive changes were even more robust. However, the most pronounced positive changes involving the reduction in edema and the restoration of retinal structure were observed after the retrobulbar administration of NCSCs.

Study limitations. A limitation of our study is the study of the effect of cell therapy only on morphological changes in the structure of the retina.

Prospects for further research. Prospective directions of further research in our opinion is to study the effect of cell therapy in glaucoma on the structure of the optic nerve, to determine the mechanisms of therapeutic action of stem cells and to study the functional parameters of the visual analyzer (for example, to determine the evoked potentials of the optic nerve).

The study opens the prospects for cell therapy in the treatment of glaucoma optic neuropathy in patients with glaucoma, which is one of the leading causes of blindness and disability worldwide today.

\section{Conclusions}

In this experiment, a positive effect of NCSCs transplantation was observed in an adrenaline-induced glaucoma model. The intravenous administration of NCSCs reduced swelling in all retinal layers but did little to restore the cytoarchitectonics of the retinal layers. After the parabulbar administration of NCSCs, there was a decrease in edema and restoration of the cytoarchitectonics of the retinal layers, most pronouncedly the retinal ganglion cell layer and in the inner retinal 
layer. However, the most pronounced reduction in edema and restoration of the cytoarchitectonics of the retinal layers was observed after the retrobulbar administration of NCSCs.

\section{Conflict of interests}

The authors declare that they have no conflicts of interest.

\section{References}

[1] Weinreb, R. N., Aung, T., Medeiros, F. A. (2014). The Pathophysiology and Treatment of Glaucoma. JAMA, 311(18), 1901-1911. doi: http://doi.org/10.1001/jama.2014.3192

[2] Wang, X., Harmon, J., Zabrieskie, N., Chen, Y., Grob, S., Williams, B. et. al. (2010). Using the Utah Population Database to assess familial risk of primary open angle glaucoma. Vision Research, 50 (23), 2391-2395. doi: http://doi.org/10.1016/ j.visres.2010.09.018

[3] Tham, Y.-C., Li, X., Wong, T. Y., Quigley, H. A., Aung, T., Cheng, C.-Y. (2014). Global Prevalence of Glaucoma and Projections of Glaucoma Burden through 2040. Ophthalmology, 121 (11), 2081-2090. doi: http://doi.org/10.1016/j.ophtha.2014.05.013

[4] Križaj D. (2019). What is glaucoma? Webvision: The Organization of the Retina and Visual System. Available at: https:// webvision.med.utah.edu/book/part-xii-cell-biology-of-retinal-degenerations/what-is-glaucoma/

[5] Mahabadi N., Foris L.A., Tripathy K. (2019). Open Angle Glaucoma. Available at: http://www.ncbi.nlm.nih.gov/ pubmed/28722917

[6] Johnson, T. V., Bull, N. D., Hunt, D. P., Marina, N., Tomarev, S. I., Martin, K. R. (2010). Neuroprotective Effects of Intravitreal Mesenchymal Stem Cell Transplantation in Experimental Glaucoma. Investigative Opthalmology \& Visual Science, 51 (4), 2051-2059. doi: http://doi.org/10.1167/iovs.09-4509

[7] Wang, Y., Xu, K., Zhang, H., Zhao, J., Zhu, X., Wang, Y., Wu, R. (2014). Retinal ganglion cell death is triggered by paraptosis via reactive oxygen species production: A brief literature review presenting a novel hypothesis in glaucoma pathology. Molecular Medicine Reports, 10 (3), 1179-1183. doi: http://doi.org/10.3892/mmr.2014.2346

[8] Elisseeff, J., Guo, Q., Lu, Q., Madrid, M., Chae, Jj. (2013). Future perspectives for regenerative medicine in ophthalmology. Middle East African Journal of Ophthalmology, 20 (1), 38-45. doi: http://doi.org/10.4103/0974-9233.106385

[9] Manuguerra-GagnÉ, R., Boulos, P. R., Ammar, A., Leblond, F. A., Krosl, G., Pichette, V. et. al. (2013). Transplantation of Mesenchymal Stem Cells Promotes Tissue Regeneration in a Glaucoma Model Through Laser-Induced Paracrine Factor Secretion and Progenitor Cell Recruitment. STEM CELLS, 31 (6), 1136-1148. doi: http://doi.org/10.1002/stem.1364

[10] Dang, Y., Zhang, C., Zhu, Y. (2015). Stem cell therapies for age-related macular degeneration: the past, present, and future. Clinical Interventions in Aging, 10, 255-264. doi: http://doi.org/10.2147/cia.s73705

[11] Roubeix, C., Godefroy, D., Mias, C., Sapienza, A., Riancho, L., Degardin, J. et. al. (2015). Intraocular pressure reduction and neuroprotection conferred by bone marrow-derived mesenchymal stem cells in an animal model of glaucoma. Stem Cell Research \& Therapy, 6 (1). doi: http://doi.org/10.1186/s13287-015-0168-0

[12] Mead, B., Hill, L. J., Blanch, R. J., Ward, K., Logan, A., Berry, M. et. al. (2016). Mesenchymal stromal cell-mediated neuroprotection and functional preservation of retinal ganglion cells in a rodent model of glaucoma. Cytotherapy, 18 (4), 487-496. doi: http://doi.org/10.1016/j.jcyt.2015.12.002

[13] Weber, M., Apostolova, G., Widera, D., Mittelbronn, M., Dechant, G., Kaltschmidt, B., Rohrer, H. (2015). Alternative Generation of CNS Neural Stem Cells and PNS Derivatives from Neural Crest-Derived Peripheral Stem Cells. Stem Cells, 33 (2), 574-588. doi: http://doi.org/10.1002/stem.1880

[14] Binder, E., Rukavina, M., Hassani, H., Weber, M., Nakatani, H., Reiff, T. et. al. (2011). Peripheral Nervous System Progenitors Can Be Reprogrammed to Produce Myelinating Oligodendrocytes and Repair Brain Lesions. Journal of Neuroscience, 31 (17), 6379-6391. doi: http://doi.org/10.1523/jneurosci.0129-11.2011

[15] Ding, S., Kumar, S., Mok, P. (2017). Cellular Reparative Mechanisms of Mesenchymal Stem Cells for Retinal Diseases. International Journal of Molecular Sciences, 18 (8), 1406. doi: http://doi.org/10.3390/ijms18081406

[16] Vasyliev, R. G., Gubar, O. S., Gordiienko, I. M., Litvinova, L. S., Rodnichenko, A. E., Shupletsova, V. V. et.al. (2019). Comparative Analysis of Biological Properties of Large-Scale Expanded Adult Neural Crest-Derived Stem Cells Isolated from Human Hair Follicle and Skin Dermis. Stem Cells International, 2019, 1-20. doi: http://doi.org/10.1155/2019/ 9640790

[17] Narytnyk, A., Verdon, B., Loughney, A., Sweeney, M., Clewes, O., Taggart, M. J., Sieber-Blum, M. (2014). Differentiation of Human Epidermal Neural Crest Stem Cells (hEPI-NCSC) into Virtually Homogenous Populations of Dopaminergic Neurons. Stem Cell Reviews and Reports, 10 (2), 316-326. doi: http://doi.org/10.1007/s12015-013-9493-9 
[18] Liu, J. A., Cheung, M. (2016). Neural crest stem cells and their potential therapeutic applications. Developmental Biology, 419 (2), 199-216. doi: http://doi.org/10.1016/j.ydbio.2016.09.006

[19] Mikheytseva, I. N. (2014). Glaucoma modeling and adrenal stress. The Journal of Clinical and Experimental Medical Research, 2 (4), 427-437. Available at: http://essuir.sumdu.edu.ua/handle/123456789/38944

[20] Vasyliev, R. G. (2014). In vitro properties of neural crest-derived multipotent stem cells from a bulge region of whisker follicle. Biotechnologia Acta, 7 (4), 71-79. doi: http://doi.org/10.15407/biotech7.04.071

\title{
PREOPERATIVE ENDOBRONCHIAL SANITATION AS PREPARATION FOR THORACIC INTERVENTIONS
}

\author{
Vasiliy Kritzak ${ }^{1}$ \\ Kritsakvv@gmail.com \\ Kateryna Ponomarova ${ }^{1}$ \\ eponkat@gmail.com \\ Dmitriy Minukhin \\ Department of surgery No. 1 \\ Kharkiv National Medical University \\ 4 Nauky ave., Kharkiv, Ukraine, 61022 \\ minukhindima@gmail.com \\ ${ }^{1}$ Department of Thoraco-Abdominal Surgery \\ V. T. Zaytsev Institute of General and Emergency Surgery of NAMS of Ukraine \\ 1 Balakireva entry, Kharkiv, Ukraine, 61018
}

\begin{abstract}
The aim - to study the effectiveness of endoscopic photodynamic therapy as a preoperative preparation of the tracheobronchial tree in patients with lung cancer.

Materials and methods. The study included 181 patients with II-III stage of lung's cancer. Diagnose of lung's cancer was confirmed with a morphologically and concomitant endobronchitis. During the preoperative preparation, we used a technique developed by us introducing into the tracheobronchial tree an aqueous solution of brilliant green at concentration of $0.04 \%$, followed by irradiation of this solution with laser radiation with the wavelength of $0.63 \mu \mathrm{m}$ (AFL-2 helium-neon laser) in an independent form and in combination with traditional anti-inflammatory therapy. Endoscopic endobronchial sanitation therapy was carried out by low-intensity radiation of the red part of the spectrum $(\lambda=0.63-0.66 \mu \mathrm{m})$ in a pulsed mode, with a power of $12 \mathrm{~mW}$.

Results. After 3-5 days of the start preoperative preparation and 1-2 sessions of endoscopic bronchosanation, the general state of patients was improved, the amount of sputum decreased and was changing from mucopurulent to mucous. To completely stop the clinical phenomena of endobronchitis, it was necessary to conduct 5-6 sessions of endoscopic photodynamic therapy according to our methodology, with the introduction of our method. In patients without pronounced clinical symptoms of concomitant chronic bronchitis, it was enough to complete only 3 sessions of bronchosanation.

Conclusion. Endoscopic photodynamic bronchosanation in the preoperative period in patients for correcting accompanying endobronchitis can significantly reduce or completely stop the clinical manifestations of endobronchitis in the shortest time and leads to a significant decrease in the number of endobronchial complications in surgical and combined treatment of lung cancer.
\end{abstract}

Keywords: preoperative preparation, endoscopic photodynamic therapy, complications. 